\title{
All-Optical Switching In Microring Resonators Using Active Optical Materials
}

\author{
Ronald Dekker, Kerstin Wörhoff, and Alfred Driessen. \\ University of Twente, Faculty of Electrical Engineering, Mathematics and Computer Science, \\ Integrated Optical Micro Systems, P.O.Box 217, 7500 AE Enschede, The Netherlands. \\ Phone: +31-53-489 4440; E-mail: R.Dekker@utwente.nl.
}

\begin{abstract}
First, the demand for high-speed active microring resonators in next generation optical networks will be explained, followed by a brief description of our preliminary results on rare earth doped nanoparticle dispersed polymers. Based on these results we propose some possible all-optical switching schemes that we will investigate using microring resonators that are made of this type of optically non-linear material.
\end{abstract}

\section{INTRODUCTION}

Microring resonators are interesting building blocks for next generation optical networks. They can be applied passively as compact filters, but as active components they are even more interesting. By utilizing the non-linear optical properties of the microring material, tunable filters and switches can be made. The speed is typically in the milliseconds and nanoseconds range for thermo-optic and electro-optic based devices respectively. Unfortunately, these speeds are insufficient for use in next generation network nodes. For the next generation optical networks, switching speeds in the Terahertz range are needed. To obtain these extremely high speeds, ultrafast physical phenomena like the third-order optical non-linearity should be exploited. The high intensity dependent refractive index of neodymium and erbium doped materials in combination with compact microring resonators could be the right choice for ultrafast switching in the second and third telecommunication window respectively.

\section{MATERIALS}

We are developing an optically non-linear hybrid material that exhibits optical gain, i.e. rare earth doped $\mathrm{LaF}_{3}$ nanoparticle dispersed photosensitive high refractive index polymers with low background absorption in the visible and infrared spectrum. By combining the relatively long excited state lifetime of rare-earth dopants in $\mathrm{LaF}_{3}$ nanoparticles [1], with the easy processing and broad range of properties of polymers, a material can be synthesized that has the best properties of both worlds. Polymers can be spinned as thin films, made photosensitive for easy patterning and nanoparticle solubility can be very high when the nanoparticle ligands are modified such that they are compatible with the polymer host. 


\section{OPTICAL GAIN}

We have measured the three dominant emission peaks at 860,863 and $866 \mathrm{~nm}$ respectively of $\mathrm{LaF}_{3}: \mathrm{Nd}$ nanoparticle dispersed PMMA which was used as a cladding material on a high contrast $\mathrm{Si}_{3} \mathrm{~N}_{4}$ single mode waveguide as function of pump power. The emission at $863 \mathrm{~nm}$ monotonously increases with increasing pump power, while the height of the emission peaks at $860 \mathrm{~nm}$ and $866 \mathrm{~nm}$ show saturation around $20 \%$ of the maximum applied pump power. This might be an indication that stimulated emission at $863 \mathrm{~nm}$ is taking place in this sample [2]. Furthermore, we have observed a small optical gain of $0.1 \mathrm{~dB} / \mathrm{cm}$ in low index contrast $\mathrm{LaF}_{3}: \mathrm{Nd}$ nanoparticle doped PMMA multimode reactively ion etched waveguides [3]. This gain can be increased by processing high contrast single mode waveguides with smooth sidewalls, increasing the rare earth dopant concentration, encapsulating the nanoparticles with silica to increase lifetime and improve the pump beam coupling to the waveguides.

\section{ALL-OPTICAL SWITCHING}

By applying the rare earth doped hybrid material with optical gain described above in combination with compact microring resonators, we are investigating 3 possible alloptical switching techniques. The first method is to tune the microring resonator in and out of resonance by inducing a refractive index change $\left(\Delta \mathrm{n} \sim 10^{-5}\right)$ making use of the intensity dependent refractive index, which is known to be high for rare earth doped, nanoparticle doped and polymer materials. Second, wavelength conversion could be used to generate 'in-resonance' wavelengths by means of Four Wave Mixing [4]. Third, cross phase modulation by strong pump pulses can induce a frequency shift on the signal wavelength and the wavelength converted signal will or will not pass a Fiber Bragg Grating [5] or in our case a high-Q microring resonator. Polymer materials with low background losses that can be reflowed to minimize sidewall roughness are promising materials for high-Q low loss microring resonator devices.

\section{ACKNOWLEDGEMENTS}

We would like to thank Wiljan Stouwdam and Mart Diemeer for their kind help with the preparation of the nanoparticles and the polymer hosts.

\section{REFERENCES}

1. J.W. Stouwdam, and F.C.J.M. van Veggel, Nano Letters, 2(7), pp733-737, 2002.

2. D.J.W. Klunder, "Photon physics in integrated optics microresonators", PhD thesis, University of Twente, Enschede, The Netherlands, 2002.

3. J.W. Stouwdam, PhD thesis, University of Twente, Enschede, The Netherlands, 2003.

4. P. Absil, J.V. Hryniewicz, B.E. Little, P.S. Cho, R.A. Wilson, L.G. Joneckis, and P.-T. Ho., Opt.Lett., 25(8), pp554-556, 2000.

5. V.E. Perlin, and H.G. Winful, IEEE Phot.Techn.Lett., 13(9), pp960-962, 2001. 